\title{
Characterization of Melt Layers in Firn at Summit, Greenland using Micro CT
}

\author{
R. W. Lomonaco, I. Baker
}

Thayer School of Engineering, Dartmouth College, 8000 Cummings Hall, Hanover, NH, 03755

Firn is multi-year snow that is not yet fully compacted into ice. It is formed by the slow densification of snow in a temperature gradient under an overburden pressure. It is of interest because its porous nature allows atmospheric air to penetrate tens of meters. With increasing depth, pores become closed off and a record of historic atmospheric compositions becomes stored within. Thus, knowing the pore structure of firn and how air moves thorough firn is key to interpreting climatic records retained in ice cores. Three melt layers exist in the Summit, Greenland firn core at $40.6 \mathrm{~m}, 46.8 \mathrm{~m}$ and $74.1 \mathrm{~m}$. Melt layers form at the surface and have a higher density and lower porosity than adjacent layers, and therefore, hinder airflow. This paper provides a brief analysis of the melt layer at $74.1 \mathrm{~m}$ using a micro CT.

A Skyscan 1172 micro CT located in a $-10^{\circ} \mathrm{C}$ cold room, operated at a $40 \mathrm{kV}$ and $250 \mu \mathrm{A}$, was used to characterize the structure of firn. A series of projection images were acquired at a step size of $0.9^{\circ}$ for an $180^{\circ}$ rotation. For this analysis an approximately $1 \mathrm{~cm}$ thick melt layer from $74.1 \mathrm{~m}$ depth was analyzed. Parameters of interest were density, structure model index (SMI) and the number and volume of pore spaces. SMI measures the convexity of the ice space, and is calculated with the use of an algorithm that artificially dilates the ice space by one voxel. It is calculated by $\mathrm{SMI}=6\left(\mathrm{~S}^{\prime} \mathrm{x} \mathrm{V}\right) / \mathrm{S}^{2}$, where $\mathrm{V}$ is the original volume of the ice matrix, $\mathrm{S}$ is the surface area before dilation, and $\mathrm{S}^{\prime}$ is the difference between the dilated and undilated surface areas. SMI has negative values for concave structures and positive values for convex structures. Values of 0,3 , and 4 relate to planar, cylindrical and spherical shapes.

Seven samples were analyzed, 3 above and 3 below the melt layer as well as one centered within the melt zone. The 3D reconstructions in Figure 1 show the pore spaces. The SMI values are much smaller within the melt layer. A background SMI value of -6 occurs at $74 \mathrm{~m}$, while within the melt layer the value was -14.6 (Fig 2a) indicating that the ice/air interface has concave curvature and that pore spaces are closed. Density values increased significantly within the melt zone (Fig. 2b). Background values at $74 \mathrm{~m}$ are $\sim 800 \mathrm{~kg} / \mathrm{m}^{3}$ but within the melt zone the density was $890 \mathrm{~kg} / \mathrm{m}^{3}$, which is close to the density of ice. The number of pores reaches a maximum at 170 within the melt layer while outside the melt layer there are only 30-50 pore spaces (Fig 3a). The distribution of the pore volume also reveals differences between the melt layer and adjacent firn. Samples outside the melt layer have a consistent quantity of pore spaces with small peaks in the $0.1-0.5 \mathrm{~mm}^{3}$ and $0.01-0.05 \mathrm{~mm}^{3}$ range (Fig. $3 \mathrm{~b}$ ). The melt layer contains a large quantity of very small pore spaces $(0.001-0.1 \mathrm{~mm} 3)$ and only two medium sized $\left(0.1-0.5 \mathrm{~mm}^{3}\right)$ pore spaces. Therefore, within the melt layer there are many small pore spaces while outside fewer larger pores exist.

As indicated by the low SMI values, a density near that of ice, and a large number of very small pore spaces, the $\sim 1 \mathrm{~cm}$ thick melt layer at $74.1 \mathrm{~m}$ may be impermeable or semi-permeable within the lock-inzone. However, in the past, when at a shallower depth, the melt layer presumably disrupted air movement within the diffusive column. Therefore, it is evident that not only the regular matrix but melt layers need to be analyzed in order to determine where air becomes isolated from the atmosphere.

This research was supported by the U.S. National Science Foundation Grant OPP 0440523. 


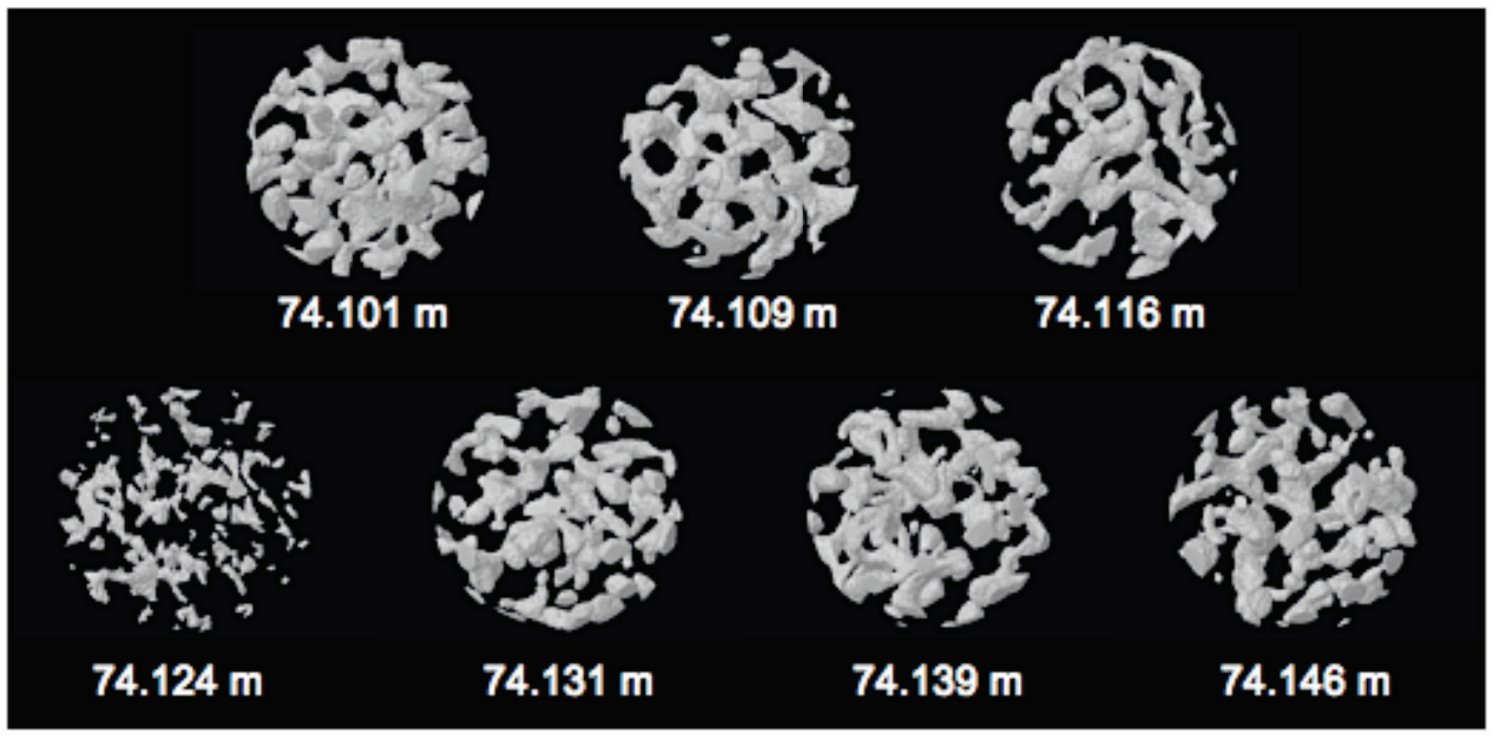

$6 \mathrm{~mm}$

Figure 1: Shows seven 3D reconstructions showing the pores from which the parameters of interest were calculated. Note that the sample labeled $74.124 \mathrm{~m}$ is the sample centered within the melt zone.
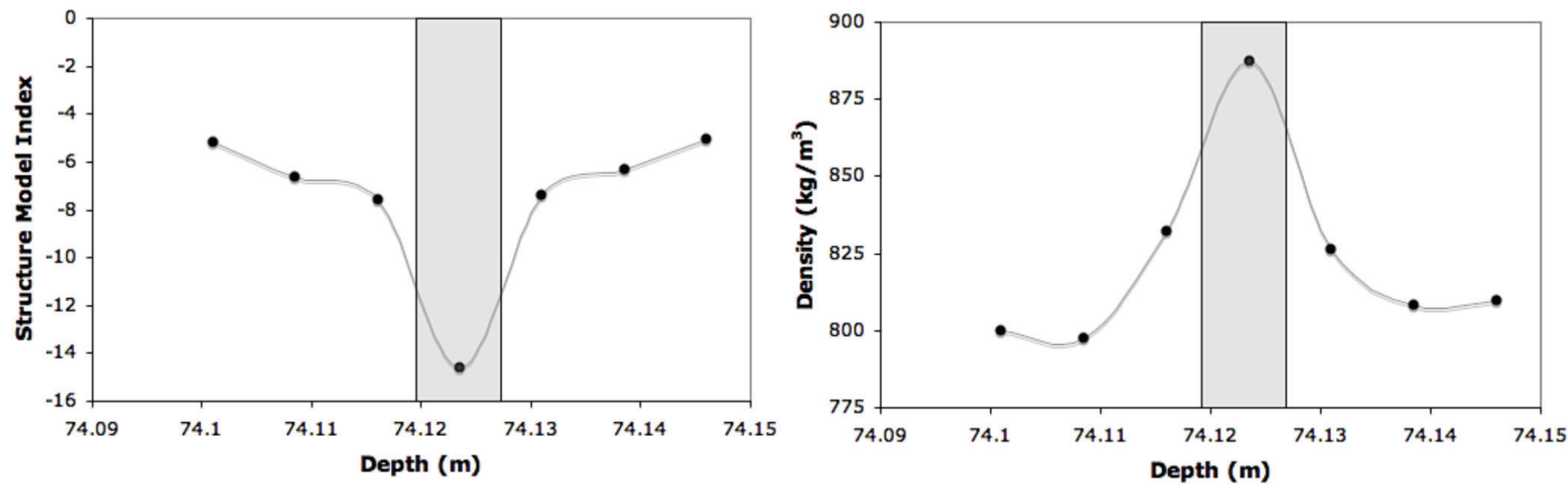

Figure 2 Graphs of (a) Structure Model Index values and (b) density with depth across the melt layer. The extent of the melt zone is shown as a gray band on the graphs.
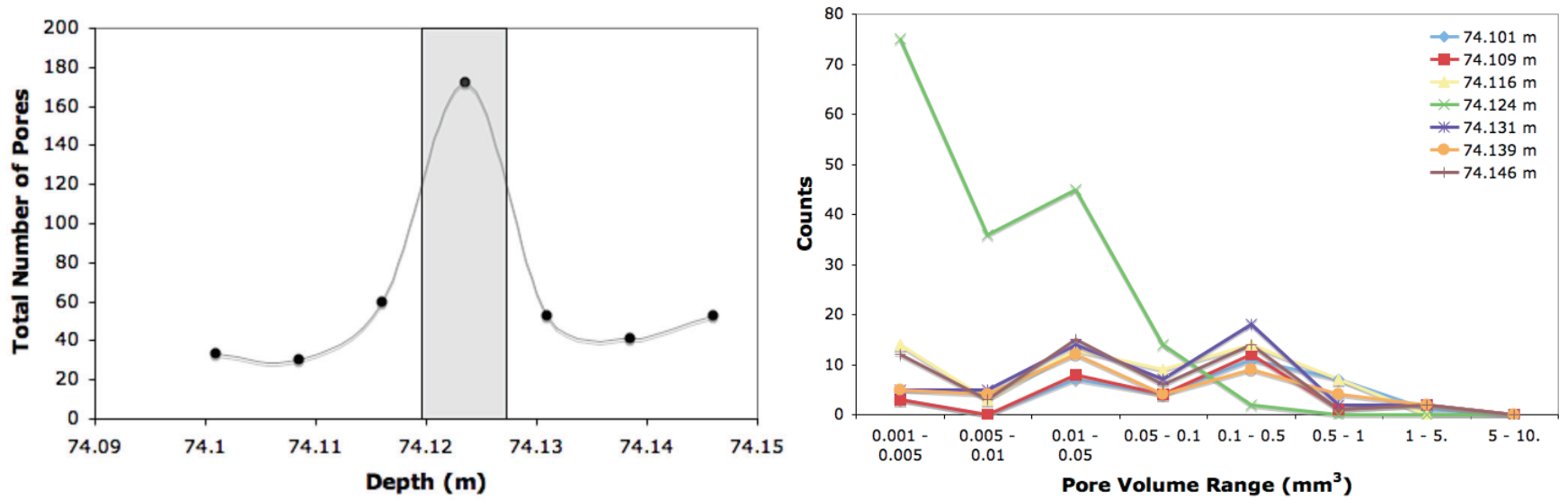

Figure 3: (a) Graph of the number of pore spaces with depth across the melt layer (the melt layer extent is represented as a gray band on the graph), and (b) distribution of pore sizes with depth. 